\title{
Talbot effect based on Raman-induced grating
}

\author{
V. G. Arkhipkin ${ }^{1,2 *}$ and S. A. Myslivets ${ }^{1,2 \dagger}$ \\ ${ }^{1}$ Kirensky Institute of Physics, Federal Research Center KSC SB RAS, 660036 Krasnoyarsk, Russia \\ ${ }^{2}$ Department of Photonics and Laser Technology, \\ Siberian Federal University, Krasnoyarsk 660041, Russia
}

(Dated:)

\begin{abstract}
We theoretically study the Talbot effect resulting from Raman-induced grating based on periodic spatial modulation of the Raman gain and dispersion in the field of a standing pump wave. Features of integer and fractional Talbot effect are demonstrated for 1D and 2D Raman-induced gratings. It is shown that the intensity of diffraction images can increase due to Raman amplification in the grating. Glass-shaped diffraction patterns are demonstrated for $2 \mathrm{D}$ gratings. It is also shown that in the vicinity of the Talbot planes there are planes in which the diffraction patterns are spatially compressed and the intensity becomes greater. The results expand the possibility of using the Talbot effect in various applications.
\end{abstract}

PACS numbers: 42.50.Gy, 42.50.Hz, 42.65.Dr, 42.79.Dj

\section{INTRODUCTION}

The Talbot effect (TE) (also referred to as selfimaging or lensless imaging) is a near-field diffraction phenomenon. The effect arises when spatially periodic structures (gratings) are illuminated by light wave $[1,2]$. TE can give self-imaging at certain periodic planes (the Talbot planes), where imaging of a grating is periodically repeated with a period $Z_{T}=2 \Lambda^{2} / \lambda$ (the Talbot's length) in the direction of light propagation $(\Lambda$ is the grating period, $\lambda$ is the wavelength of the illuminating light). This effect is also called the integer Talbot effect. At the distance $Z(p / q) Z_{T}$ ( $p$ and $q$ are positive integer, $p<q$ ), diffractive patterns are similar to the grating but the period of the image is different from that of the object. This property is referred to as the fractional Talbot effect (FTE). These phenomena are the consequences of interference between diffraction orders, which acquire a quadratic phase under Fresnel propagation. These effects have interesting applications in many different areas, for example, in optical imaging and computing [1], optical microscopy [3], Talbot array illumination [4, 5], and lithography [6]. The Talbot effect has also been demonstrated in atomic waves [7], Bose-Einstein condensates [8], waveguide arrays [9], plasmonic Talbot effect [10], exciton polaritons [11], and metamaterials [12]. In addition, the Talbot effect has been extended to the temporal Talbot effect [13], nonlinear Talbot effect [14], angular Talbot effect [15], and PT-symmetric Talbot effect [16].

In most of the existing works the grating to be imaged is usually material. Recently, the Talbot effect was reported using a nonmaterial grating [17-19]. This grating is an electromagnetically induced grating (EIG) [20]. It is based on electromagnetically induced transparency (EIT) phenomenon [21]. Such an induced grating leads

\footnotetext{
*avg@iph.krasn.ru

† sam@iph.krasn.ru
}

to self-images, which are called the electromagnetically induced Talbot effect (EITE). EITE offers a nondestructive and lensless way of imaging ultracold atoms and molecules in the Fresnel diffraction region [17]. It possesses certain distinguishable features due to the lightinduced atomic coherence in atomic media and allows easy control of different experimental parameters in order to get an optimal configuration for desired applications. An atomic imaging scheme based on the second-order two-photon EITE is proposed in [22].

Another type of a lensless imaging scheme based on electromagnetically induced holographic imaging is proposed in [23]. In contrast to the EITE scheme [17], this one allows both the amplitude and phase information of the generated EIG to be imaged with the characteristic of the arbitrarily controllable image variation in size.

In this paper, we propose another type of EITE which is based on a Raman-induced grating (RIG) in atomic media [24-26]. Such a grating occurs during Raman interaction of the probe field with a standing-wave pump field in atomic media. Unlike EIGs based on EIT, RIG is based on spatial modulation of the Raman gain and refractive index for the probe wave in a pump standingwave. In the paper [27], a Fraunhofer diffraction was investigated, when the probe field propagates normal to the standing wave, and RIG can operate as a diffraction grating. Herein, we study the features of integer and fractional Talbot effect from one (1D) - and two (2D) dimensional Raman-induced gratings. These phenomena are observed in Fresnel diffraction in the near field and are fundamentally different from the Fraunhofer diffraction in the far field. This work may expand the variety of applications in imaging techniques and be useful for optical lithography as well.

\section{MODEL}

We consider a homogeneously broadened medium consisting of an ensemble of three-level atoms in a $\Lambda$ con- 
(a)

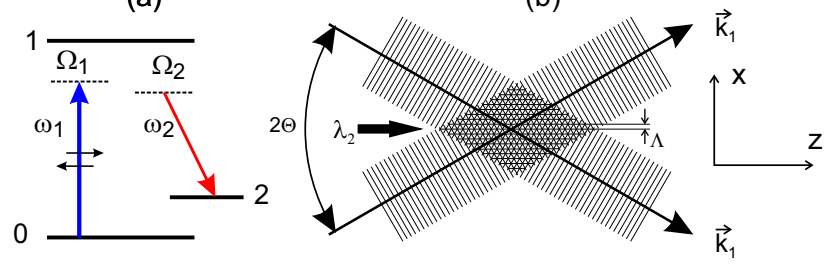

FIG. 1. (a) A three-level $\Lambda$-type atomic system for Raman induced grating. (b) Configuration of a standing-wave pump field.

figuration, with two metastable lower states $|0\rangle$ and $|2\rangle$ (Fig. 1a). Initially, the entire population is distributed in the ground state $|0\rangle$. Two ground states $|0\rangle$ and $|2\rangle$ are coupled to the excited state $|1\rangle$ via a standing-wave pump field of angular frequency $\omega_{1}$ with a large detuning $\Omega_{1}$, and a weak probe field of angular frequency $\omega_{2}$ with a detuning $\Omega_{2}$. In the case of a $1 \mathrm{D}$ grating the pump field consists of two fields that propagate at an angle $2 \Theta$ symmetrically with respect to $z$ direction and, when intersecting generate a standing wave within the medium along the $x$ direction with a spatial period $\Lambda=\lambda_{1} /[2 \sin \Theta]$, depending on the angle $\Theta$ (Fig. 1b). The probe field propagates along the $z$ direction normal to the standing wave. For a $2 \mathrm{D}$ grating the pump field is to be a combination of two orthogonal standing-waves of the same frequency, and the probe field propagates normal to the the $x-y$ plane in intersection region of the two orthogonal standing pump waves. We first consider a $1 D$ grating. In the approximation of a thin grating [20], when diffraction within the bulk of the medium can be ignored, the probe field $E_{2}(x, L)$ at the output surface $z=L$ is

$$
E_{2}(x, L)=E_{2}(z=0) \exp \left(-k_{2} \chi_{2}^{\prime \prime} L\right) \exp \left(i k_{2} \chi_{2}^{\prime} L\right),
$$

Here $E_{2}(0)$ is the amplitude of the probe plane-wave at the input surface, $L$ is the medium length, $k_{2}=\omega_{2} / c$, $\chi_{2}^{\prime}=\operatorname{Re} \chi_{2}, \chi_{2}^{\prime \prime}=\operatorname{Im} \chi_{2}$.

The linear macroscopic susceptibility $\chi_{2}\left(\omega_{2}\right)$ of the system at the probe frequency $\omega_{2}$ is expressed as [27]

$$
\chi_{2}\left(\omega_{2}\right)=\alpha_{r} \frac{\gamma_{12}}{\Omega_{1}^{2}} \frac{\left|G_{p}\right|^{2}}{\left(\Omega_{20}+i \gamma_{20}+\left|G_{p}\right|^{2} / \Omega_{1}\right)}
$$

$\alpha_{r}=\left|d_{12}\right|^{2} N / 2 \hbar \gamma_{12}, G_{p}(x)=G_{1} \sin (\pi x / \Lambda)$ is the Rabi frequency of the pump field, $\Omega_{1,2}=\omega_{1,2}-\omega_{10,12}$ is onephoton detuning, $\Omega_{20}=\Omega_{1}-\Omega_{2}$ is the Raman detuning, $\omega_{m n}, \gamma_{m n}$ and $d_{m n}$ are the frequency, half width, and matrix dipole moment of transition, respectively; $\hbar$ is the Plank constant. Near the Raman resonance $\left(\left|\Omega_{20}\right| \ll\left|\Omega_{1}\right|,\left|\Omega_{2}\right|\right)$ the detuning $\Omega_{2} \approx \Omega_{1}$. Here we assume that $\left|\Omega_{1}\right| \gg \gamma_{10}, G_{1}$ and that the probe field is weak considering that all atoms remain in the ground state $|0\rangle$ in the process of interaction. It can be seen from (2) that by changing the intensity and frequency of the pump field, one can effectively control the susceptibility $\chi_{2}\left(\omega_{2}\right)$. When $\left|G_{p}\right|^{2} /\left|\Omega_{1}\right|>\gamma_{20}$ the pump field
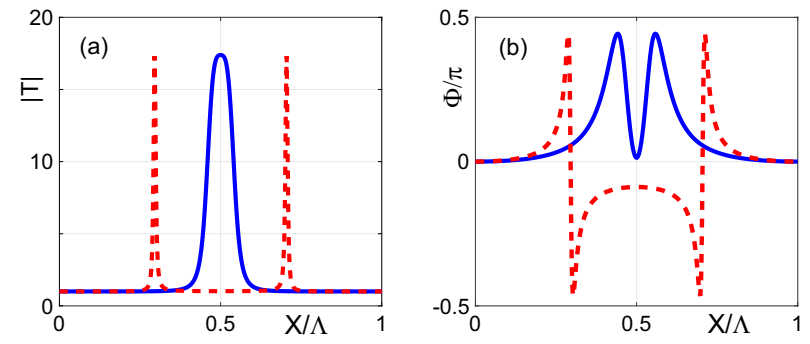

FIG. 2. Output profile of the probe field amplitude $|T(x, L)|$ (a) and the phase $\Phi(x, L)$ (b) as a function of $x$ within a single space period. The parameters are $G_{1}=2$ (solid curves) and $G_{1}=2.5$ (dashed curves), $\Omega_{20}=40$.

induces an ac-Stark shift of the state $|1\rangle$, which leads to a shift of the Raman resonance by $\Omega_{S}=\left|G_{p}\right|^{2} / \Omega_{1}$ depending on the transverse coordinates. Since the Stark shift is proportional to $\left|G_{1}\right|^{2}$, then the amplitude of perturbed resonance increases significantly in comparison with the unperturbed one. From (2) and (1), it can be seen that the Raman gain and the refractive index for the probe field are periodically modulated in a space along the direction $x$.

\section{RESULTS AND DISCUSSION}

In our calculation we used the parameters for D1 line of sodium atoms and levels $|0\rangle$ and $|2\rangle$ correspond to the long-lived superfine sublevels of the ground state ${ }^{2} S_{1 / 2}$. The following atomic parameters are used: $\gamma_{10} / 2 \pi=10$ $\mathrm{MHz}, \gamma_{21}=\gamma_{10}, \gamma_{20}=10^{-3} \gamma_{10}$. The Rabi frequency $G_{1}$ and one-photon detuning $\delta_{1}$ are given in $\gamma_{10}$ units, the Raman detuning $\Omega_{20}$ in the units $\gamma_{20}, \Omega_{1}=-100$, the atomic media length $L=10$ is given in the units $z_{0}=1 / k_{2} \alpha_{r}$, and the grating period is $\Lambda=20 \lambda_{1}$. Let's introduce the complex transmission (gain) function $T(x, L)=E_{2}(x, L) / E_{2}(z=0)=|T(x, L)| \exp (i \Phi(x, L)$, where $|T(x, L)|=\exp \left(-k_{2} \chi_{2}^{\prime \prime} L\right)$ is the amplitude and $\Phi(x, L)=\exp \left(i k_{2} \chi_{2}^{\prime} L\right)$ is phase of the transmission function. Typical plots of $|T(x, L)|$ and $\Phi(x, L)$ as a function of $x$ are shown in Fig. 2. It is clear that the probe field is amplified and the gain depends on the transverse coordinate $x$ and reiterates along $x$ with a period $\Lambda$ controlled by the standing pump wave. Thus, an optical grating (non-material) is induced on the output plane on which the probe wave diffracts. This grating is a combination of an amplitude (gain) and a phase (refraction) gratings, that is hybrid one. The period of the grating $\Lambda$ can be made arbitrarily larger than the wavelength $\lambda_{2}$ of the probe field by varying the angle between the two pump fields generating a standing wave. The grating profile on the x-coordinate depends on the standing wave amplitude $G_{1}$ and Raman detuning $\Omega_{20}$. The transmission profile represents one or two symmetrical peaks, which is due to the ac-Stark shift of the Raman resonance induced 

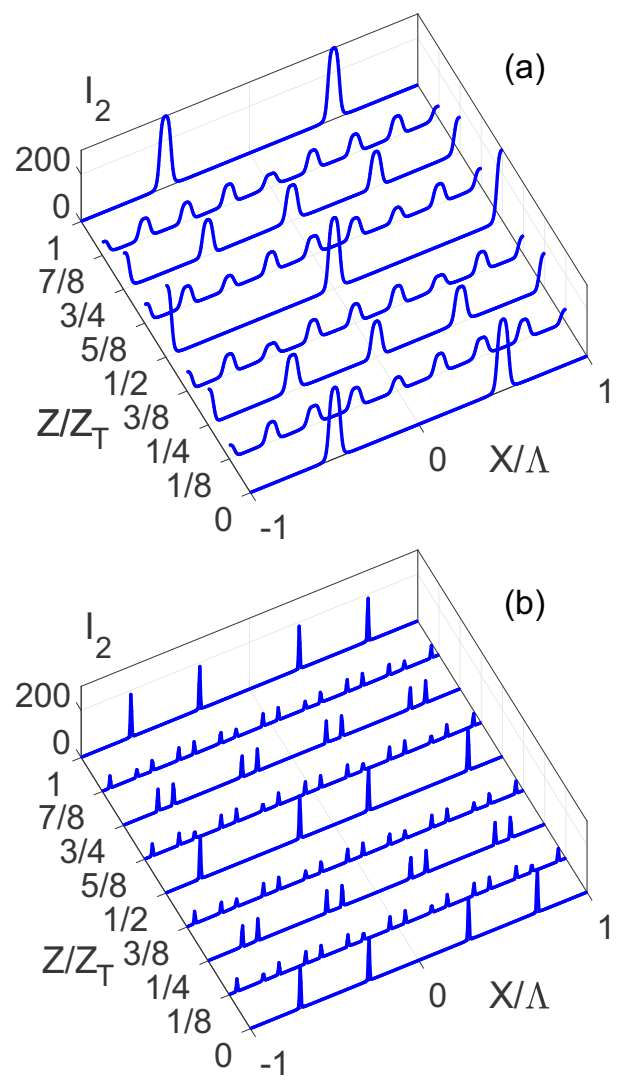

FIG. 3. Intensity distributions of the probe field at various distances within a two-space period. The parameters are $G_{1}=2(a), G_{1}=2.5(b)$ and $\Omega_{20}=40$.

by the pump field (Fig. 2a). The position of the peaks corresponds to the perturbed Raman resonance (with the account of the Stark shift). The solid blue line in Fig. 2a corresponds to the case when a perturbed Raman resonance occurs for the pump field at the center of the period.

With the increasing pump field, a spatial splitting of the peak occurs (dashed red line), and the larger the $G_{1}$, the stronger the peaks shift and they are spatially compressed. The phase of the transmission function is also spatially modulated along the $x$ direction (Fig. $2 \mathrm{~b}$ ).

Using the Fresnel-Kirchhoff diffraction integral under the paraxial approximation [28], the output probe field $E_{2}$ for a $1 D$ grating at distance $Z$ from the output surface of the medium can be expressed as

$$
\begin{aligned}
& E_{2}(X, Z)= \\
& \quad \frac{1+i}{\sqrt{2 \lambda_{2} Z}} e^{-i k_{2} Z} \int_{-\infty}^{\infty} T(x, L) e^{-\frac{i k_{2}}{2 Z}(x-X)^{2}} d x,
\end{aligned}
$$

where $x$ and $X$ are the coordinates in the object and observation planes, respectively.

Completing the integral in (3) with the Fourier series
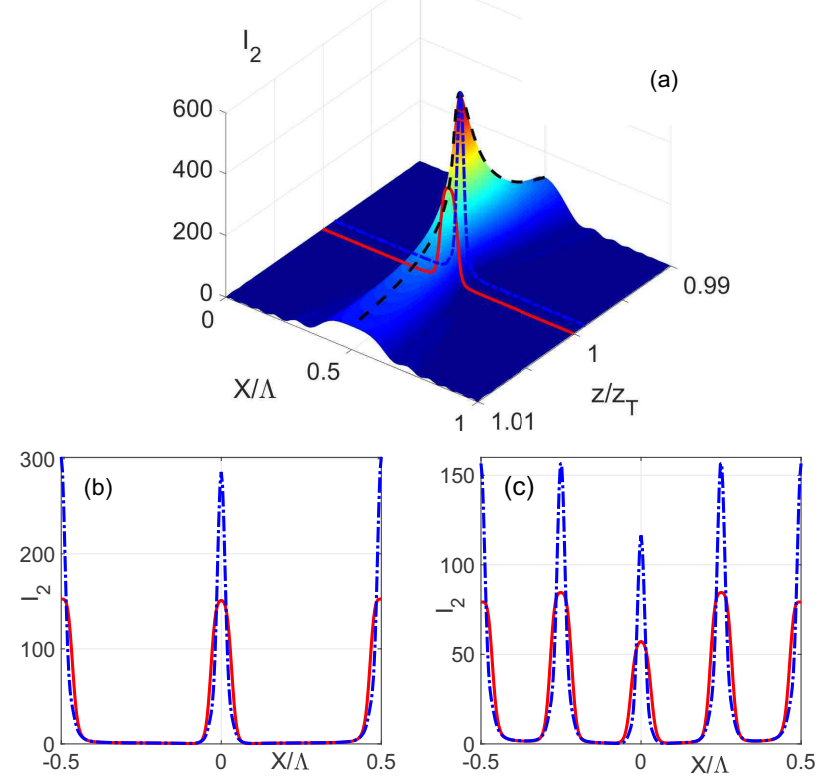

FIG. 4. (a) Intensity distribution of the diffraction pattern as a function of $X$ and $Z$ near the Talbot plane $Z=Z_{T}$ within a single-space period. The solid curve (red) corresponds to the intensity distribution in the Talbot plane, the dash-dotted (blue) line corresponds to the maximum intensity plane. $(b, c)$ Intensity distribution $I_{2}(X)$ in the planes of maximum amplitude, at a distance of $a=-10^{-3} Z_{T}$ from the planes $Z=Z_{T} / 4$ (b) and $Z=Z_{T} / 8$ (c), (dash-dotted blue curves). For comparison, an intensity profile is shown in $Z=Z_{T} / 4$ (b) and $Z=Z_{T} / 8$ (c) (solid red curves). The parameters are $G_{1}=2$ and $\Omega_{20}=40$.

expansion of $T(x, L)$, we can obtain the Talbot effect as

$$
\begin{aligned}
& E_{2}(X, Z)= \\
& \quad i e^{-i k_{2} Z} \sum_{n} C_{n} \exp \left(i 2 \pi n X / \Lambda-i 2 \pi n^{2} Z / Z_{T}\right),
\end{aligned}
$$

where $Z_{T}=2 \Lambda^{2} / \lambda_{2}$ is the Talbot length, $C_{n}$ - is the Fourier coefficient (or the amplitude of the n-th harmonic).

From equation (4), we can see the typical features of the Talbot effect on a RIG. At a certain distance $Z=m Z_{T}$, where $m$ denotes a positive integer, the field amplitude matches the amplitude at the output plane of the RIG (the self-images) and all diffraction orders are in phase. For $Z=Z_{T} / 2$ the field amplitude is exactly the same as the amplitude at the output RIG plane, but the phase is shifted by half a period. Figure 3 shows typical transverse profiles of a diffracted wave at distances $Z=(p / 8) Z_{T}(p=0,1,2 \ldots 8)$ from the grating. It can be seen that their intensity can be substantially higher than the intensity of the radiation incident on the medium. This is due to the Raman gain in the grating. For fractional $Z_{T}$, the field amplitude and the period undergo change and the diffraction pattern is no longer a replica 
(self-image) of the grating, but it is periodic in nature. With fractional values of $Z_{T}$, the period changes, and the intensity distribution is no longer a RIG self-image. Note that in Fig. $3 \mathrm{~b}$ the number of peaks in one period is twice the number in Fig. 3a. This is due to splitting of the transmission function $\mathrm{T}$ (Fig. 2b) caused by the Stark effect.

Figure 4a shows intensity distribution of the diffraction pattern on $X$ and $Z$ coordinates near the Talbot plane $Z=Z_{T}$. It can be seen that there is a plane $Z<Z_{T}$ (dash-dotted curve), where the maximum intensity is greater than in the Talbot plane (solid curve), and spatial distribution on the transverse coordinate becomes narrower, that is, spatial compression occurs. This behavior takes place as for integer and fractional TE (Fig. 4b,c). Thus, near the Talbot planes there are planes that can be called the planes of maximum intensity.

To understand this result, introduce the notation $Z=$ $Z_{T}-a$, where $a$ is the distance from the $Z_{T}$ plane to the maximum intensity plane, and rewrite formula (4) as

$$
E_{2}(X, a) \sim \sum_{n} C_{n} \exp \left(i 2 \pi n X / \Lambda+i 2 \pi n^{2} a / Z_{T}\right)
$$

Formula (5) for $E_{2}(X, Z)$ in the plane $Z=Z_{T}-a$ differs from the field in the Talbot plane by the additional phase $2 \pi n^{2} a / Z_{T}$, which can renormalize the phases of the harmonics in such a way that spatial compression of the diffraction patterns occurs, as can be seen from Fig. 4.

Consider now the case of a $2 \mathrm{D}$ grating. In this case the pump field is formed by two standing waves that are directed along the $x$ and $y$ axes (perpendicular to each other) $E_{p}(x, y)=E_{1}\left[\sin \left(\pi x / \Lambda_{x}\right)+\sin \left(\pi y / \Lambda_{y}\right)\right]$, where $\Lambda_{x, y}$ is the period of a standing wave along axes $x$ and $y$, respectively. The probe field propagates along the $z$ direction perpendicular to the $x-y$ plane. In this case, the transmission function $T(x, y, L)$ will be periodic in $x$ and $y$ directions $T(x, y, L)=T\left(x+2 \Lambda_{x}, y, L\right)=$ $T\left(x, y+2 \Lambda_{y}, L\right)$ with the period $2 \Lambda_{x, y}$. Figure 5 a,b shows two typical profiles of the transmission function for a $2 \mathrm{D}$ grating on the output face of the medium $|T(x, y, L)|^{2}$. The parameter $G_{1}$ is chosen such that there is no spatial splitting in $T(x, y, L)$, in the former case (Fig. 5a) whereas in the latter case, (Fig. 5b) $T(x, y, L)$ splits. In the former case, the intensity distribution has one peak structure, and in the latter case, it is glass-shaped. It can be seen that for the $2 D$ grating, the period in the $x$ and $y$ directions has doubled compared to the $1 \mathrm{D}$ grating.

For a $2 \mathrm{D}$ grating the probe field $E_{2}$ at a distance $Z$ from the grating is

$$
\begin{aligned}
& E_{2}(X, Y, Z)= \\
& \frac{i}{\lambda_{2} Z} e^{-i k_{2} Z} \int_{-\infty}^{\infty} T(x, y, L) e^{-\frac{i k_{2}}{2 Z}\left[(x-X)^{2}+(y-Y)^{2}\right]} d x d y .
\end{aligned}
$$

Expanding $T(x, y, L)$ in a Fourier series, yields the diffracted amplitude $E_{2}(X, Y, Z)$ in the form

$$
\begin{aligned}
& E_{2}(X, Y, Z)= \\
& e^{-i k_{2} Z} \sum_{n, m} C_{n, m} \exp \left(i 2 \pi n X / \Lambda_{1 x}+i 2 \pi m Y / \Lambda_{1 y}\right) \\
& \quad \exp \left(-i \pi n^{2} \lambda_{2} Z / \Lambda_{1 x}^{2}-i \pi m^{2} \lambda_{2} Z / \Lambda_{1 y}^{2}\right) .
\end{aligned}
$$

Here $\Lambda_{1 x, 1 y}=2 \Lambda_{x, y}$ is the spatial period of $T(x, y, L)$ along directions $x$ and $y$, respectively.

Note that 2D diffraction grating cannot be considered as the superposition of two 1D gratings in contrast to conventional material gratings. This is due to the fact that the transmission of the RIG is a nonlinear function of the pump field, depending on the $x$ and $y$ coordinates, and therefore cannot be represented as a product of functions that depend only on one coordinate.

From (6), we can see that ET is observed for two cases. The first case is $\Lambda_{1 x}=\Lambda_{1 y}=\Lambda_{1}$ and the second one is $\Lambda_{1 x} / \Lambda_{1 y}=a / b$, where $a$ and $b$ are mutually prime integers. The distance at which self-imaging is observed is called the Talbot length. In the former case the Talbot length is $Z_{T}=2 \Lambda_{1}^{2} / \lambda_{2}$. In the latter case, when $\Lambda_{1 x} / \Lambda_{1 y}=a / b$, the Talbot length is $Z_{T}=2 q^{2} \Lambda_{x}^{2} / \lambda_{2}=2 p^{2} \Lambda_{y}^{2} / \lambda_{2}$. Under these conditions all diffraction orders are in phase and the object function is
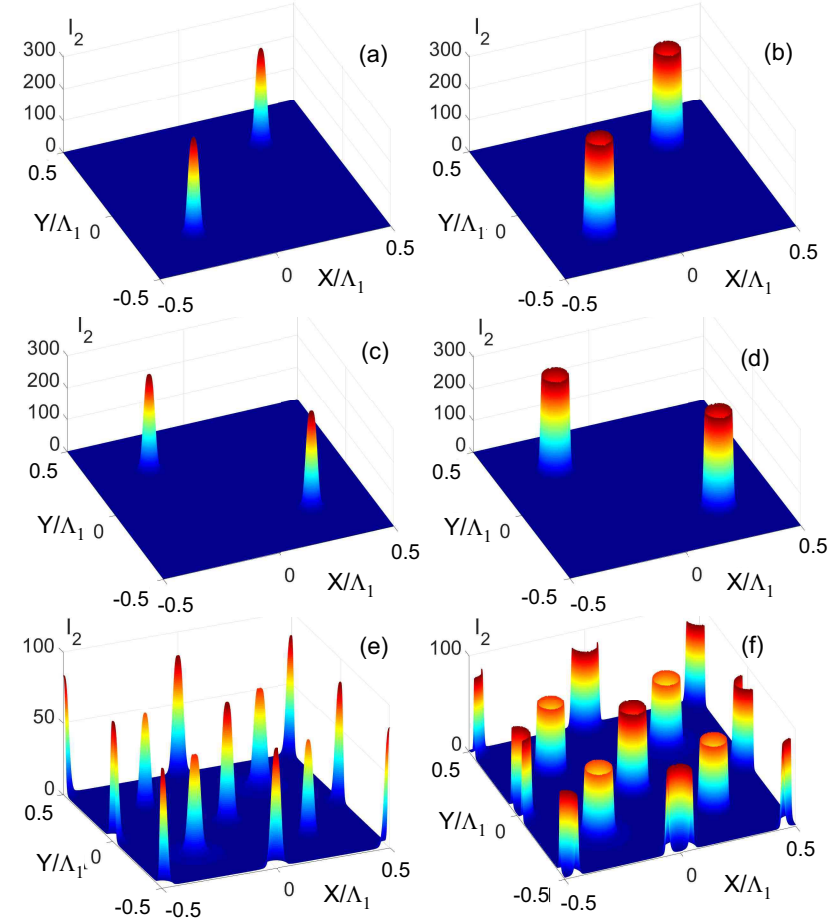

FIG. 5. Intensity distribution of the diffraction patterns as a function of $X$ and $Y$ within a single-space period at the distances $Z=Z_{T}$ and $Z=Z_{T} / 2$ (a), (b), $Z=Z_{T} / 4$ (c), (d) and $Z=Z_{T} / 8$ (e), (f) for $G_{1}=1$ (a,c,e), and $G_{1}=1.03$ $(\mathrm{b}, \mathrm{d}, \mathrm{f})$. 
reproduced. From (6) it follows that

$$
E_{2}(X, Y, Z) \simeq \sum_{n, m} C_{n, m} \exp \left[-2 i \pi\left(b^{2} n^{2}+a^{2} m^{2}\right) Z / Z_{T}\right]
$$

The analysis shows that the Fourier coefficients $C_{n, m}$ are nonzero only if $n$ and $m$ have the same parity. Then two options are possible: 1 ) if $a$ and $b$ have different parity, the factor $\left(b^{2} n^{2}+a^{2} m^{2}\right)$ is always odd, and 2) $a$ and $b$ are odd, then $\left(b^{2} n^{2}+a^{2} m^{2}\right)$ is always even. It follows that in the first case, the field distribution is similar to the 1D case, that is, for $Z=Z_{T}$, the field distribution exactly coincides with the field distribution on the output plane of the RIG, is shifted by half the period for $Z=Z_{T} / 2$, has a period of two times smaller for $Z=Z_{T} / 4$, and so on. In the second case, in the $Z=Z_{T}$ and $Z=Z_{T} / 2$ planes, the field distribution coincides with that on the output plane of the RIG, as shown in Fig. 5a,b, (for the case of $a=b=1$ ), and is shifted by half the period in the plane $Z=Z_{T} / 4$, as shown in fig. $5 \mathrm{c}, \mathrm{d}$. Figure $5 \mathrm{e}, \mathrm{f}$ shows Talbot images in the $Z=Z_{T} / 8$ plane. Thus, the behavior of diffraction patterns for fractional ET in $2 \mathrm{D}$ gratings is fundamentally different from 1D case.

\section{CONCLUSION}

In conclusion, we have studied, for the first time to the best of our knowledge, the features of integer and fractional Talbot effect on 1D and 2D Raman-induced gratings in different conditions and shown how to control the diffraction pattern in the Talbot planes. In contrast to conventional gratings, the intensity of diffraction patterns (Talbot images) under certain conditions can be enhanced by Raman amplification in the grating. It is shown that near the Talbot planes there are planes in which the intensity is greater than in the Talbot planes, and the degree of their spatial localization is greater. It is shown that single-peak and glass-shaped Talbot images can be obtained on a two-dimensional grating. The predicted effects are the result of the combined action of the amplitude and phase gratings. The dependence of the field distribution in the Talbot fractional planes for the $2 \mathrm{D}$ grating on the ratio of the periods along the $x$ and $y$ coordinates is determined. The results obtained may be of interest for microscopy using the Talbot effect, Talbot array illumination, photolithography and etc.

\section{ACKNOWLEDGMENTS}

This work was supported by the Russian Science Foundation (RSF) through Grant 19-12-00203.
[1] K. Patorski, The self-imaging phenomenon and its applications (Elsevier, 1989) Chap. 1, pp. $1-108$.

[2] J. Wen, Y. Zhang, and M. Xiao, The talbot effect: recent advances in classical optics, nonlinear optics, and quantum optics, Adv. Opt. Photon. 5, 83 (2013).

[3] S. Feng, M. Wang, and J. Wu, Lensless in-line holographic microscope with talbot grating illumination, Opt. Lett. 41, 3157 (2016).

[4] A. W. Lohmann and J. A. Thomas, Making an array illuminator based on the talbot effect, Appl. Opt. 29, 4337 (1990).

[5] C. R. Fernández-Pousa, R. Maram, and J. Azaña, Cwto-pulse conversion using temporal talbot array illuminators, Opt. Lett. 42, 2427 (2017).

[6] Q. Wu, H. Xia, H. Jia, H. Wang, C. Jiang, L. Wang, J. Zhao, R. Tai, S. Xiao, D. Zhang, S. Yang, and J. Jiang, Fast and large-area fabrication of plasmonic reflection color filters by achromatic talbot lithography, Opt. Lett. 44, 1031 (2019).

[7] S. Wu, E. Su, and M. Prentiss, Demonstration of an areaenclosing guided-atom interferometer for rotation sensing, Phys. Rev. Lett. 99, 173201 (2007).

[8] C. Ryu, M. F. Andersen, A. Vaziri, M. B. d'Arcy, J. M. Grossman, K. Helmerson, and W. D. Phillips, High-order quantum resonances observed in a periodically kicked bose-einstein condensate, Phys. Rev. Lett. 96, 160403 (2006).

[9] R. Iwanow, D. A. May-Arrioja, D. N. Christodoulides, G. I. Stegeman, Y. Min, and W. Sohler, Discrete talbot effect in waveguide arrays, Phys. Rev. Lett. 95, 053902 (2005).

[10] W. Zhang, C. Zhao, J. Wang, and J. Zhang, An experimental study of the plasmonic talbot effect, Opt. Express 17, 19757 (2009).

[11] T. Gao, E. Estrecho, G. Li, O. A. Egorov, X. Ma, K. Winkler, M. Kamp, C. Schneider, S. Höfling, A. G. Truscott, and E. A. Ostrovskaya, Talbot effect for exciton polaritons, Phys. Rev. Lett. 117, 097403 (2016).

[12] H. Nikkhah, M. Hasan, and T. J. Hall, The talbot effect in a metamaterial, Applied Physics A 124, 106 (2018).

[13] L. Deng, E. W. Hagley, J. Denschlag, J. E. Simsarian, M. Edwards, C. W. Clark, K. Helmerson, S. L. Rolston, and W. D. Phillips, Temporal, matter-wave-dispersion talbot effect, Phys. Rev. Lett. 83, 5407 (1999).

[14] X.-B. Song, H.-B. Wang, J. Xiong, K. Wang, X. Zhang, K.-H. Luo, and L.-A. Wu, Experimental observation of quantum talbot effects, Phys. Rev. Lett. 107, 033902 (2011).

[15] J. Azaña and H. Guillet de Chatellus, Angular talbot effect, Phys. Rev. Lett. 112, 213902 (2014).

[16] H. Ramezani, D. N. Christodoulides, V. Kovanis, I. Vitebskiy, and T. Kottos, $\mathcal{P} t$-symmetric talbot effects, Phys. Rev. Lett. 109, 033902 (2012).

[17] J. Wen, S. Du, H. Chen, and M. Xiao, Electromagnetically induced talbot effect, Applied Physics Letters 98, 081108 (2011), https://doi.org/10.1063/1.3559610.

[18] J. Sheng, J. Wang, M.-A. Miri, D. N. Christodoulides, and M. Xiao, Observation of discrete diffraction patterns 
in an optically induced lattice, Opt. Express 23, 19777 (2015).

[19] J. Yuan, C. Wu, Y. Li, L. Wang, Y. Zhang, L. Xiao, and S. Jia, Integer and fractional electromagnetically induced talbot effects in a ladder-type coherent atomic system, Opt. Express 27, 92 (2019).

[20] H. Y. Ling, Y.-Q. Li, and M. Xiao, Electromagnetically induced grating: Homogeneously broadened medium, Phys. Rev. A 57, 1338 (1998).

[21] M. Fleischhauer, A. Imamoglu, and J. P. Marangos, Electromagnetically induced transparency: Optics in coherent media, Rev. Mod. Phys. 77, 633 (2005).

[22] T. Qiu, G. Yang, and Q. Bian, Electromagnetically induced second-order talbot effect, EPL (Europhysics Letters) 101, 44004 (2013).

[23] T. Qiu, L. Xia, H. Ma, C. Zheng, and L. Chen, Electromagnetically induced holographic imaging, Optics Com- munications 358, 20 (2016).

[24] V. G. Arkhipkin and S. A. Myslivets, Raman-induced gratings in atomic media, Opt. Lett. 39, 3223 (2014).

[25] V. G. Arkhipkin and S. A. Myslivets, Coherent manipulation of the raman-induced gratings in atomic media, Phys. Rev. A 93, 013810 (2016).

[26] V. G. Arkhipkin and S. A. Myslivets, Coherent control of light-pulse propagation in a raman induced grating, J. Opt. 19, 055501 (2017).

[27] V. G. Arkhipkin and S. A. Myslivets, One- and twodimensional raman-induced diffraction gratings in atomic media, Phys. Rev. A 98, 013838 (2018).

[28] K. Iizuka, Engineering optics (Springer-Verlag New York, 2008) p. 532, 3rd ed. 